\title{
Non-standard interactions using the OPERA experiment
}

\author{
Mattias Blennow $^{1, \mathrm{a}}$, Davide Meloni ${ }^{2, \mathrm{~b}}$, Tommy Ohlsson ${ }^{3, \mathrm{c}}$, Francesco Terranova ${ }^{4, \mathrm{~d}}$, Mattias Westerberg ${ }^{3, \mathrm{e}}$ \\ ${ }^{1}$ Max-Planck-Institut für Physik (Werner-Heisenberg-Institut), Föhringer Ring 6, 80805 Munich, Germany \\ ${ }^{2}$ Dipartimento di Fisica, Universitá di Roma Tre and INFN Sez. di Roma Tre, Via della Vasca Navale 84, 00146 Rome, Italy \\ ${ }^{3}$ Department of Theoretical Physics, School of Engineering Sciences, Royal Institute of Technology-AlbaNova University Center, \\ Roslagstullsbacken 21, 10691 Stockholm, Sweden \\ ${ }^{4}$ Laboratori Nazionali di Frascati dell'INFN, Via E. Fermi 40, 00044 Frascati, Italy
}

Received: 25 April 2008 / Published online: 7 August 2008

(C) Springer-Verlag / Società Italiana di Fisica 2008

\begin{abstract}
We investigate the implications of non-standard interactions on neutrino oscillations in the OPERA experiment. In particular, we study the non-standard interaction parameter $\varepsilon_{\mu \tau}$. We show that the OPERA experiment has a unique opportunity to reduce the allowed region for this parameter compared with other experiments such as the MINOS experiment, mostly due to the higher neutrino energies in the CNGS beam compared to the NuMI beam. We find that OPERA is mainly sensitive to a combination of standard and non-standard parameters and that a resulting anti-resonance effect could suppress the expected number of events. Furthermore, we show that running OPERA for five years each with neutrinos and anti-neutrinos would help in resolving the degeneracy between the standard parameters and $\varepsilon_{\mu \tau}$. This scenario is significantly better than the scenario with a simple doubling of the statistics by running with neutrinos for ten years.
\end{abstract}

\section{Introduction}

Neutrino oscillation physics has definitively entered the era of precision measurements of the fundamental neutrino parameters such as the neutrino mass squared differences (i.e., $\Delta m_{31}^{2}$ and $\Delta m_{21}^{2}$ ) and the leptonic mixing parameters (i.e., $\theta_{12}, \theta_{13}, \theta_{23}$, and $\delta$ ). In particular, the Super-Kamiokande, SNO, KamLAND, K2K, and MINOS experiments have given valuable information on these parameters [1-6].

\footnotetext{
a e-mail: blennow@mppmu.mpg.de

b e-mail: meloni@ fis.uniroma3.it

c e-mail: tommy@theophys.kth.se

d e-mail: Francesco.Terranova@cern.ch

e e-mail: mwesterb@kth.se
}

The precision measurements open up the possibility to investigate if neutrino flavor transitions are governed by neutrino oscillations only or if they are, in the next-to-leading order, a combination of neutrino oscillations and some other new physics mechanism. However, to leading order, there exists clear evidence that neutrino oscillations constitute the underlying physical model for neutrino flavor transitions. The next-to-leading order mechanism could e.g. be nonstandard interactions (NSIs), mass varying neutrinos, neutrino decay, neutrino decoherence etc., or some combination thereof.

In this work, we will study NSI effects at the OPERA experiment [7], which is an experiment that consists of a massive lead/emulsion target (the OPERA detector) located at LNGS in Gran Sasso, Italy, receiving its neutrino beam, originally consisting almost exclusively of $v_{\mu}$, from CERN in Geneva, Switzerland. The baseline length is approximately $732 \mathrm{~km}$ and the CNGS $v_{\mu}$ beam has an average neutrino energy of $E_{v} \simeq 17 \mathrm{GeV}$. The OPERA experiment is especially designed to observe $v_{\tau}$ events from the $v_{\mu} \rightarrow v_{\tau}$ neutrino oscillation channel. In fact, no previous experiment has investigated this channel or observed neutrinos of a different flavor than that originally produced at the source (although the neutral-current measurements at SNO imply that solar $v_{e}$ have oscillated into a different flavor). Thus, the OPERA experiment presents a unique opportunity to study the direct appearance of $v_{\tau}$ [8]. In this work, we will not try to describe the origin of the NSIs but adopt a purely phenomenological point of view. In particular, NSIs may modify the production, the propagation in matter as well as the detection of the neutrinos. We will concentrate on the simplified scenario in which NSIs only affect the neutrino propagation.

Previously, investigations of NSIs that are of importance for this work have been presented in the following papers. In [9], a two-flavor neutrino analysis of the so-called atmospheric neutrino anomaly has been performed, which 
effectively bounds the NSI parameters in the $\mu-\tau$ sector, $\varepsilon_{\mu \tau} \simeq \varepsilon$ and $\varepsilon_{\tau \tau} \simeq \varepsilon^{\prime}$, to $-0.03 \leq \varepsilon \leq 0.02$ and $\left|\varepsilon^{\prime}\right| \leq 0.05$ at $99.73 \%$ confidence level. Although these bounds may seem quite restrictive, it has been shown that at least the bound on $\varepsilon_{\tau \tau}$ is severely weakened when considering the full three-flavor framework (allowing $\varepsilon_{\tau \tau}$ to be of $\mathcal{O}(1)$ or larger, depending on the values of $\varepsilon_{e e}$ and $\varepsilon_{e \tau}$ [10]). As will be shown later in this work, the limit that could be put by the OPERA experiment would be insensitive to whether the two- or three-flavor scenario is studied, simply because of the relatively short baseline. In addition, in [11], the authors have come to the conclusion that it would be possible to observe NSI effects at the OPERA experiment (and the ICARUS experiment) if $\varepsilon_{\mu \tau} \geq \mathcal{O}\left(10^{-2}\right)$. Next, in [12], the Kamioka-Korea two detector setup has been investigated, which could also give restrictions on the NSI parameters $\varepsilon_{\mu \tau}$ and $\varepsilon_{\tau \tau}$. Recently, in [13], a study of the OPERA experiment (in combination with the MINOS experiment) has been presented with the conclusion that it is not very sensitive to the NSI parameters $\varepsilon_{e \tau}$ and $\varepsilon_{\tau \tau}$. However, it was found that the $v_{\tau}$ sample is too small to be statistically significant to improve the limits on the NSI parameter $\varepsilon_{\tau \tau}$. Nevertheless, this analysis did not include a study of the relevant $\varepsilon_{\mu \tau}$ which, due to the energies and the baseline involved in the OPERA experiment, is the only NSI parameter appearing to leading order in $L$ in the $v_{\mu} \rightarrow v_{\tau}$ flavor transition.

In general, neutrino oscillations and NSIs in terrestrial neutrino experiments have been studied extensively in the literature, using the neutrino factory project [14-21] and other different neutrino facilities (like super-beams and $\beta$-beams) [22-26] to assess the impact of the NSI effects in neutrino physics.

This work is organized as follows. In Sect. 2, we will present analytic considerations for the NSIs that we assume for the OPERA experiment. In addition, we will comment on a sort of anti-resonance effect that is in the vicinity of being detectable in the OPERA experiment. Next, in Sect. 3, we will give the numerical setup with the GLoBES software $[27,28]$ that we use for our simulations of the OPERA experiment. Then, in Sect. 4, we will show our numerical results for the OPERA experiment using GLoBES. Finally, in Sect. 5, we will present a summary of the work as well as our conclusions.

\section{Analytic considerations}

We consider effective non-standard interactions of the form

$$
\begin{aligned}
\mathcal{L}_{\mathrm{NSI}}= & -\frac{G_{F}}{\sqrt{2}} \sum_{\substack{f=u, d, e \\
a= \pm 1}} \varepsilon_{\alpha \beta}^{f a}\left[\bar{f} \gamma^{\mu}\left(1+a \gamma^{5}\right) f\right] \\
& \times\left[\overline{v_{\alpha}} \gamma_{\mu}\left(1-\gamma^{5}\right) v_{\beta}\right],
\end{aligned}
$$

where $f$ is summed over the matter constituents, and the parameters $\varepsilon_{\alpha \beta}^{f a}$, which are the entries of a Hermitian matrix $\varepsilon^{f a}$, give the strength of the NSIs. In a manner completely analogous to the derivation of the normal matter effect, these interactions will result in an effective addition

$H_{\mathrm{NSI}}=V\left(\begin{array}{lll}\varepsilon_{e e} & \varepsilon_{e \mu} & \varepsilon_{e \tau} \\ \varepsilon_{e \mu}^{*} & \varepsilon_{\mu \mu} & \varepsilon_{\mu \tau} \\ \varepsilon_{e \tau}^{*} & \varepsilon_{\mu \tau}^{*} & \varepsilon_{\tau \tau}\end{array}\right)$

to the neutrino oscillation Hamiltonian in flavor basis, where $\varepsilon_{\alpha \beta}=\sum_{f, a} \varepsilon_{\alpha \beta}^{f a} N_{f} / N_{e}$ and $V=\sqrt{2} G_{F} N_{e}$. Notice that, apart from the bounds on $\varepsilon_{\mu \tau}$ and $\varepsilon_{\tau \tau}$ given in Sect. 1, we are not aware of any paper discussing direct bounds on the effective parameters $\varepsilon_{\alpha \beta}$. However, experimental limits on the parameters $\varepsilon_{\alpha \beta}^{f a}$ can be found in [29, 30], which imply that $\left|\varepsilon_{e \mu}^{f a}\right| \leq \mathcal{O}\left(10^{-4}\right)$ and $\left|\varepsilon_{e e}^{f a}\right| \leq \mathcal{O}(1)$ [20]. Thus, we may assume that the effective parameters $\varepsilon_{e e}$ and $\varepsilon_{e \mu}$ are bounded at the same order of magnitude as their corresponding parameters $\left|\varepsilon_{\alpha \beta}^{f a}\right|$.

The full three-flavor Hamiltonian describing neutrino propagation in matter is given by

$$
\begin{aligned}
H= & \frac{1}{2 E} U \operatorname{diag}\left(0, \Delta m_{21}^{2}, \Delta m_{31}^{2}\right) U^{\dagger} \\
& +H_{\mathrm{MSW}}+H_{\mathrm{NSI}},
\end{aligned}
$$

where $U$ is the leptonic mixing matrix, $\Delta m_{i j}^{2}=m_{i}^{2}-$ $m_{j}^{2}$, and $H_{\mathrm{MSW}}$ is the addition from the standard matter effect. Due to the quite large neutrino energy, $E_{v}=$ $\mathcal{O}(10) \mathrm{GeV}$, and the relatively short baseline, $L \simeq 732 \mathrm{~km}$, both $\Delta m_{31}^{2} L /\left(2 E_{v}\right) \ll 1$ and $V L \ll 1$, where $V$ is the matter potential; $V \simeq 1.1 \times 10^{-13} \mathrm{eV}$ in the Earth's crust $\left(\rho \simeq 2.7 \mathrm{~g} / \mathrm{cm}^{3}\right)$ [31]. Thus, neutrino oscillations will not have time to fully develop. As a consequence, the main characteristics of the flavor transition probabilities will be given by truncating the flavor evolution matrix $S=\exp (-\mathrm{i} H L)$ at order $L$, resulting in

$S \simeq \mathbb{1}-\mathrm{i} H L$.

The off-diagonal neutrino transition probabilities are then given by

$P_{\alpha \beta}=\left|S_{\beta \alpha}\right|^{2} \simeq\left|H_{\beta \alpha} L\right|^{2}$.

The diagonal neutrino survival probabilities in this expansion are given by the unitarity condition $P_{\alpha \alpha}=1-$ $\sum_{\beta \neq \alpha} P_{\alpha \beta}$. As can be observed from this consideration, the transition probabilities will only be affected by the corresponding NSI element (i.e., $P_{\alpha \beta}$ just depends on the NSI element $\varepsilon_{\beta \alpha}$ ), while the survival probabilities depend on the two off-diagonal NSI elements associated with the flavor (e.g., $P_{\mu \mu}$ is affected by $\varepsilon_{e \mu}$ and $\varepsilon_{\mu \tau}$ ). As expected, the diagonal NSI parameters do not enter at short baselines. Clearly, 
this is not true in general and at higher orders in $L$, where the NSI parameters will enter all of the neutrino oscillation probabilities. As an example, the NSI parameter $\varepsilon_{e \tau}$ will enter the flavor evolution matrix $S_{\mu \tau}$ at $\mathcal{O}\left(L^{2}\right)$ and then to $\mathcal{O}\left(L^{3}\right)$ in the transition probability $P_{\mu \tau}$ (unless there is no interference between the $L$ and $L^{2}$ terms). From the above consideration we can conclude that the NSI parameter of most interest for the OPERA experiment is $\varepsilon_{\mu \tau}$. That the parameters $\varepsilon_{e \tau}$ and $\varepsilon_{\tau \tau}$ are not important has been already shown in [13].

The main physics goal of the OPERA experiment is to actually observe oscillations of $v_{\mu}$ into $v_{\tau}$. With the effects of $\varepsilon_{\mu \tau}$ included, the transition probability $P_{\mu \tau}$ is given by

$$
\begin{aligned}
P_{\mu \tau} & =\left|S_{\tau \mu}\right|^{2} \\
& =\left|c_{13}^{2} \sin \left(2 \theta_{23}\right) \frac{\Delta m_{31}^{2}}{4 E_{v}}+\varepsilon_{\mu \tau}^{*} V\right|^{2} L^{2}+\mathcal{O}\left(L^{3}\right),
\end{aligned}
$$

where we have neglected the small mass squared difference $\Delta m_{21}^{2}$. From this consideration follows that there is a degeneracy between the standard neutrino oscillation parameters and the NSI parameter $\varepsilon_{\mu \tau}$, as scenarios with the same value of $\left|c_{13}^{2} \sin \left(2 \theta_{23}\right) \Delta m_{31}^{2} /\left(4 E_{v}\right)+\varepsilon_{\mu \tau}^{*} V\right|$ will lead to the same neutrino oscillation probability. Even if the degeneracy is broken by the energy dependence of the first term, we still expect some parameter correlations when analyzing the outcome of an experiment. It is also interesting to note that the $\mathcal{O}\left(L^{2}\right)$ contribution to $P_{\mu \tau}$ vanishes when

$\varepsilon_{\mu \tau}^{*}=-c_{13}^{2} \frac{\Delta m_{31}^{2}}{4 E_{v} V} \sin \left(2 \theta_{23}\right)$

simply due to the fact that $S_{\tau \mu}=0$ in this case. The condition clearly shows that this can happen only for real $\varepsilon_{\mu \tau}$. We will use the term anti-resonance to refer to this scenario as it, in some sense, is the opposite of the MSW-resonance: in the standard picture of neutrino oscillations, the matter effects cancel the difference between the diagonal terms and the effective mixing angle is maximal, whereas in the situation with NSIs, the matter effects cancel the off-diagonal terms and the effective mixing angle is minimal (i.e., zero). In a pure two-flavor scenario, the anti-resonance is valid to all orders, while transitions can be induced to higher order in $L$ by other off-diagonal elements in the case of three-flavor oscillations. For the peak energy of $E_{v} \simeq 17 \mathrm{GeV}$ in the CNGS beam, the anti-resonance would occur for $\varepsilon_{\mu \tau} \simeq-0.3$, with the result that no $v_{\tau}$ events would be observed. Note that a similar conclusion applies in the case of an inverted mass hierarchy, from which $\varepsilon_{\mu \tau} \simeq+0.3$ if $\Delta m_{31}^{2} \rightarrow-\Delta m_{31}^{2}$ (neglecting the small effect of $\Delta m_{21}^{2}$ ). This also applies to the case of anti-neutrinos, where we have $V \rightarrow-V$ and $\varepsilon_{\alpha \beta} \rightarrow \varepsilon_{\alpha \beta}^{*}$. In both cases, this also gives an estimate of the order of magnitude of the NSIs that OPERA will be sensitive to, as the expected number of $v_{\tau}$ events is low.
Finally, we want to mention that a similar effect could exist in the $v_{\mu} \rightarrow v_{e}$ transition. In fact,

$$
\begin{aligned}
P_{\mu e}= & \left|S_{e \mu}\right|^{2} \\
= & \mid\left[s_{23} \sin \left(2 \theta_{13}\right) \mathrm{e}^{\mathrm{i} \delta}+\alpha c_{23} c_{13} \sin \left(2 \theta_{12}\right)\right] \frac{\Delta m_{31}^{2}}{4 E_{v}} \\
& +\left.\varepsilon_{e \mu}^{*} V\right|^{2} L^{2}+\mathcal{O}\left(L^{3}\right),
\end{aligned}
$$

where $\delta$ is the standard $C P$-violating phase in the unitary leptonic mixing matrix, $\alpha=\Delta m_{21}^{2} / \Delta m_{31}^{2}$ is the ratio between the mass squared differences, and we have neglected a term proportional to $s_{13} \alpha$. In this case, the external bounds on $\varepsilon_{e \mu}$ are so stringent that the term proportional to $\alpha$ is known to be larger. Thus, an anti-resonance in this channel could only be due to an interplay between the two standard terms if $\delta=\pi$.

\section{Numerical setup}

The numerical simulations of the OPERA experiment were performed using the GLoBES software [27, 28], which was extended in order to accommodate the inclusion of NSIs through the Hamiltonian presented in (2) with $\varepsilon_{e e}=$ $\varepsilon_{e \mu}=\varepsilon_{e \tau}=0$. The neutrino propagation in matter was then described using the full three-flavor Hamiltonian in (3). In addition, the Abstract Experiment Definition Language (AEDL) file, used to describe the OPERA experiment, was based on the results presented in [7, 32, 33]. Unless stated otherwise, we have assumed a running time of five years with $4.5 \times 10^{19}$ protons on target per year, in accordance with the OPERA experimental setup, and an effective mass of 1.65 kton [7]. Furthermore, the neutral- and chargedcurrent cross-sections were taken from [7, 34, 35]. The CNGS neutrino spectra are substantially different from zero in the interval between $1 \mathrm{GeV}$ and $30 \mathrm{GeV}$ (with a peak around $E_{v} \simeq 17 \mathrm{GeV}$ ). Thus, we divided the signals and the corresponding backgrounds into 29 equally spaced energy bins, having checked that the numerical results are stable if the number of energy bins is above the order of 10 . For the baseline length of the CNGS setup (approximately $732 \mathrm{~km}$ ), the matter density profile was assumed to be constant and equal to the value at the Earth's crust, i.e., $\rho=2.72 \mathrm{~g} / \mathrm{cm}^{3}$ (or $V=1 / 1900 \mathrm{~km}^{-1}$ ) [36]. In all simulations, we have used a full three-flavor neutrino framework with central values and $1 \sigma$ errors of the standard neutrino oscillation parameters as given in Table 1. Normal mass hierarchy, i.e., $\Delta m_{31}^{2}>0$, has been assumed if not stated otherwise.

Regarding the NSI parameters, we performed numerical simulations with different simulated values, also taking into account the effects of possible $C P$-violating phases of the 
Table 1 The simulated values of the standard neutrino oscillation parameters and the corresponding $1 \sigma$ priors used in the simulations. The central values of the parameters $\theta_{12}$ and $\Delta m_{21}^{2}$ were inspired by the results of the KamLAND experiment [6], whereas the central values of the other parameters were inspired by [37]. We fixed the value of the $C P$-violating phase to $\pi / 2$, with no consequences on our results for the $v_{\mu} \rightarrow v_{\tau}$ channel

\begin{tabular}{ll}
\hline$\theta_{12}=34.4^{\circ} \pm 1.7^{\circ}$ & $\Delta m_{21}^{2}=(7.59 \pm 0.21) \times 10^{-5} \mathrm{eV}^{2}$ \\
$\theta_{13}=4.8^{\circ} \pm 2.9^{\circ}$ & $\Delta m_{31}^{2}=(2.4 \pm 0.15) \times 10^{-3} \mathrm{eV}^{2}$ \\
$\theta_{23}=45^{\circ} \pm 3.8^{\circ}$ & $\delta=\pi / 2$ \\
\hline
\end{tabular}

non-diagonal entries of the Hamiltonian in (2). The priors set on the NSI parameters are chosen according to [29], except from $\varepsilon_{\tau \tau}$, which has further been constrained using atmospheric neutrino data [10]. For comparison, we also included the MINOS experiment, which offers the possibility to probe the $v_{\mu} \rightarrow v_{e}$ transition channel, in our simulations. As already mentioned in [13], different $L / E_{v}$ could in general be very useful in order to further constrain some of the parameters of (2), since the relative importance of the standard and non-standard parts of the Hamiltonian is energy dependent. Our numerical setup of the MINOS experiment follows that used in [24].

\section{Numerical results}

In this section, we present the numerical results on the physics reach of the OPERA experiment in constraining the new physics parameters $\varepsilon_{\alpha \beta}$. In all figures, we have combined both the $v_{\mu} \rightarrow v_{e}$ and $v_{\mu} \rightarrow v_{\tau}$ channels for the OPERA experiment.
The results have been obtained by marginalizing over the parameters $\Delta m_{31}^{2}$ and $\theta_{23}$ (if not stated otherwise), while keeping the parameters $\Delta m_{21}^{2}$ and $\theta_{12}$ fixed, since they are irrelevant for the $v_{\mu} \rightarrow v_{\tau}$ transition in the OPERA experiment. In addition, the parameter $\theta_{13}$ was fixed, since it does not affect the results. We also observed that $\varepsilon_{e e}, \varepsilon_{e \mu}, \varepsilon_{\mu \mu}$, and $\varepsilon_{e \tau}$ do not affect the results, which means that they are fixed to zero in the rest of the work.

First, in Fig. 1, we present the sensitivity reach for $\varepsilon_{\mu \tau}$ with the OPERA experiment in combination with the MINOS experiment (for a discussion on the sensitivity reach for $\varepsilon_{e \tau}$ and $\varepsilon_{\tau \tau}$ for the same combination, see [13]). As can be observed in this figure, OPERA is far more sensitive to $\varepsilon_{\mu \tau}$ due to the higher neutrino energy than that in MINOS, which can therefore only marginally improve the sensitivity. Thus, in the following we will only consider the bounds which can be placed from OPERA itself.

Note that, as expected, a similar situation is also valid when considering the detection of the $v_{\mu} \rightarrow v_{e}$ transition in OPERA. In fact, as already stressed in Sect. $2, \varepsilon_{\mu \tau}$ appears to leading order in $P_{\mu \tau}$ (6), but it is subleading in $P_{\mu e}$ (8). We verified that the inclusion of the $v_{\mu} \rightarrow v_{e}$ channel does not affect the results on $\varepsilon_{\mu \tau}$.

\subsection{Marginalization of $\varepsilon_{\tau \tau}$}

Figure 2 shows the OPERA sensitivity in the $\left|\varepsilon_{\mu \tau}\right|-\varepsilon_{\tau \tau}$ plane (left panel) as well as the impact on the $\varepsilon_{\mu \tau}$ sensitivity given different priors on $\varepsilon_{\tau \tau}$ (right panel). As expected, the impact of $\varepsilon_{\tau \tau}$ is small as long as any reasonable prior is put. This is naturally related to the fact that $\varepsilon_{\tau \tau}$ enters only in higher order in the oscillation probability $P_{\mu \tau}$. From the left
Fig. 1 Sensitivity for $\varepsilon_{\mu \tau}$ at $95 \%$ confidence level (2 d.o.f.) of the OPERA and MINOS experiments in the case of no NSIs (the input values of the various $\varepsilon_{\alpha \beta}=0$ )

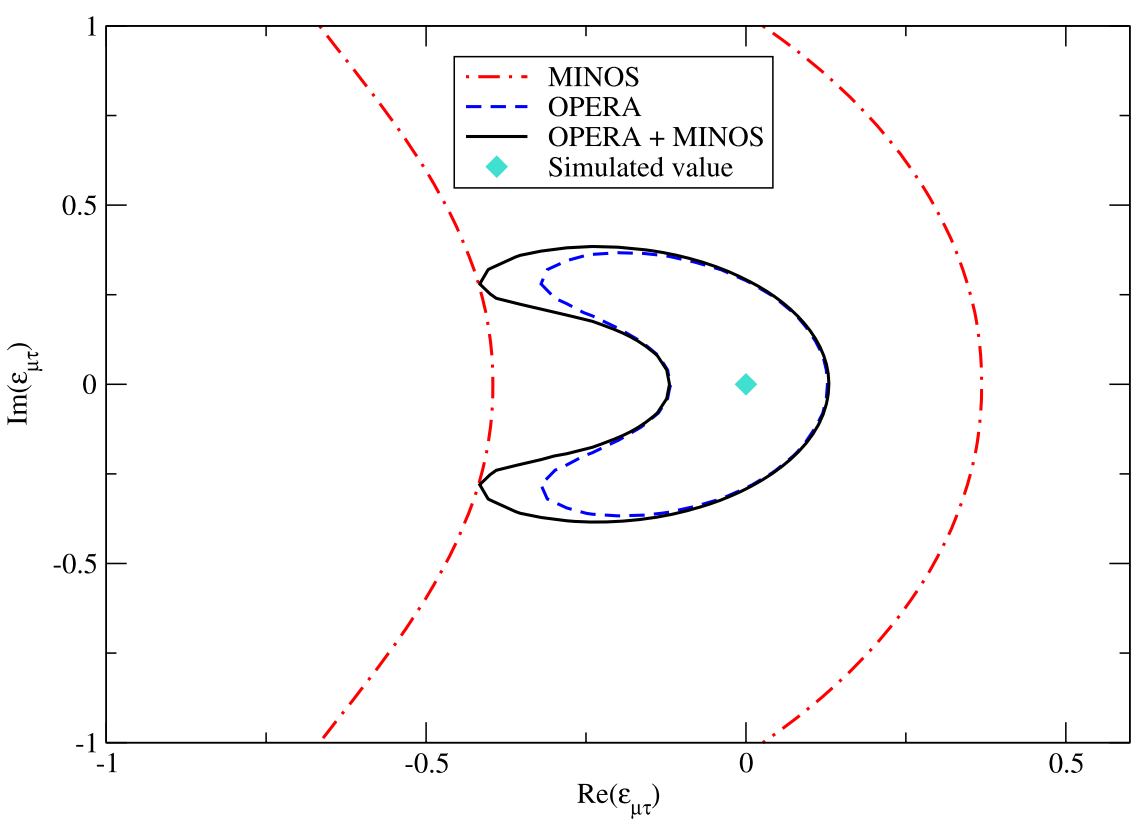



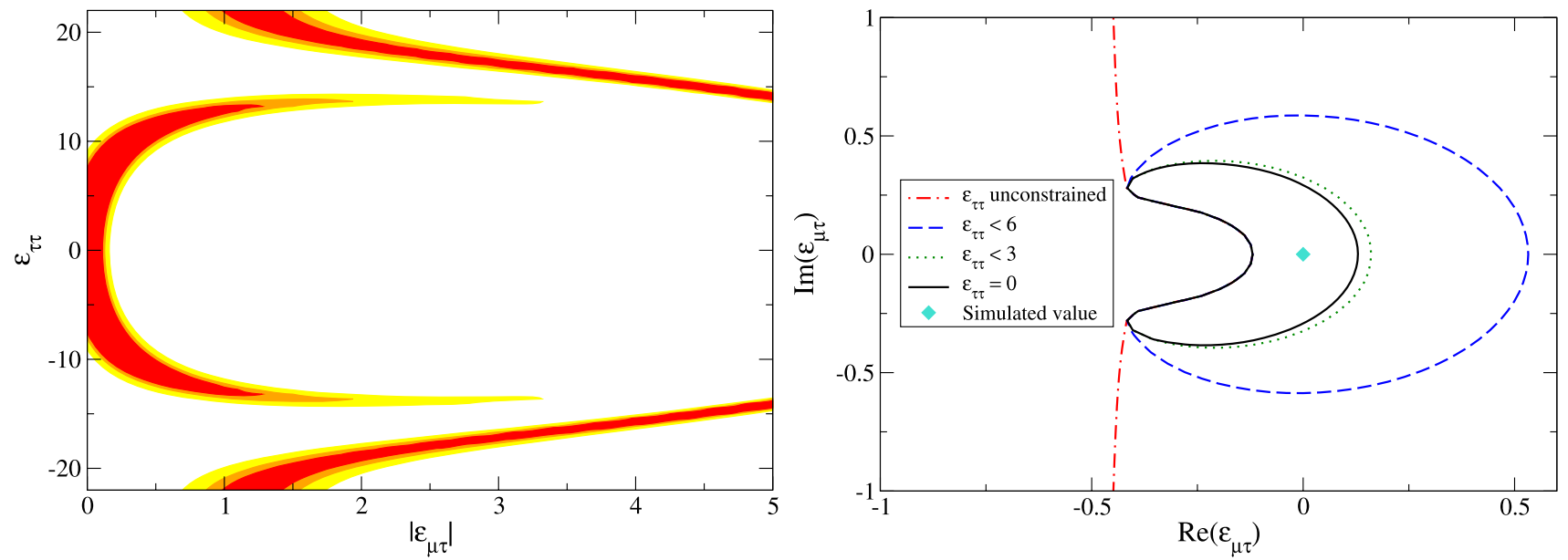

Fig. 2 The left panel shows the NSI sensitivity of the OPERA experiment alone for marginalized standard neutrino oscillation parameters. The confidence levels ( 2 d.o.f.) are $90 \%, 95 \%$, and $99 \%$, respectively. The sensitivity contours for fixed standard neutrino oscillation parameters only differ slightly from this result. The right panel shows how the sensitivity to $\varepsilon_{\mu \tau}$ changes depending on the $\varepsilon_{\tau \tau}$ prior. The sensitivity contours in the right panel are at $95 \%$ confidence level ( 2 d.o.f.) and the $\varepsilon_{\tau \tau}$ priors are at $1 \sigma$ level

Fig. 3 (Color online) The sensitivity of OPERA to the NSI parameter $\varepsilon_{\mu \tau}$ (2 d.o.f.) for five (colored regions) and ten (curves) years of running time. The simulated value of $\varepsilon_{\mu \tau}$ is zero and the confidence levels are $90 \%, 95 \%$, and $99 \%$, respectively. The diamond corresponds to the simulated value of $\varepsilon_{\mu \tau}=0$

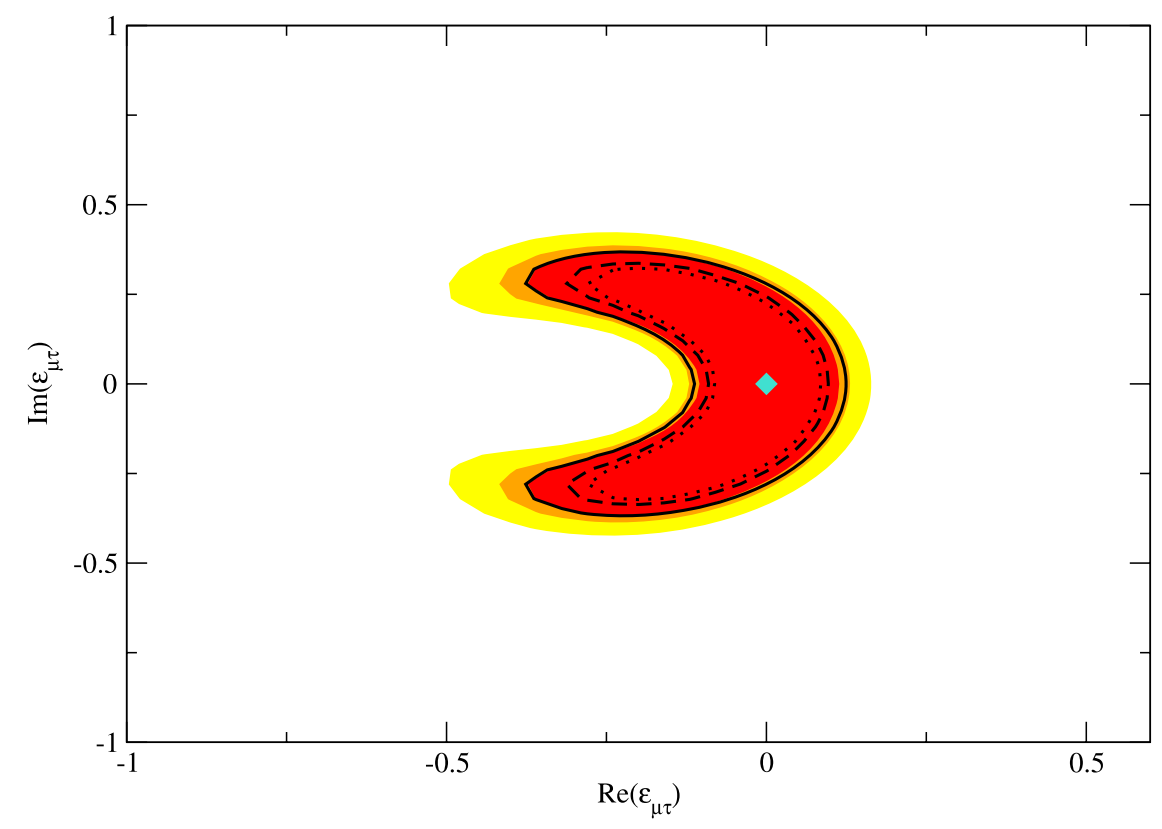

panel of the figure, it is evident that $\varepsilon_{\tau \tau}$ has to be of $\mathcal{O}(10)$ to significantly alter the prediction for $\varepsilon_{\mu \tau}$. In the rest of this work (including Fig. 1), the prior put on $\varepsilon_{\tau \tau}$ is $\left|\varepsilon_{\tau \tau}\right|<1.9$ $(1 \sigma$ confidence level).

\subsection{Sensitivity to $\varepsilon_{\mu \tau}$}

In Fig. 3, we show the predicted sensitivity of OPERA to the NSI parameter $\varepsilon_{\mu \tau}$. It is clear from this figure that the sensitivity contours extend in the direction in which the number of $v_{\tau}$ events is constant (basically a circle centered at $\varepsilon_{\mu \tau} \simeq-0.3$ ). Furthermore, the sensitivity change on running the experiment for a longer time has been indicated. As can be seen, this would slightly improve the projected sensitivity. However, running the experiment with reversed polarity could significantly improve the sensitivity; see Fig. 4. Due to the change of $V \rightarrow-V$ and $\varepsilon_{\alpha \beta} \rightarrow \varepsilon_{\alpha \beta}^{*}$ when considering anti-neutrinos instead of neutrinos, it follows from (6) that the sensitivity contours for the reversed polarity will extend in a direction different from those of the original polarity as can be observed in the figure. Thus, the combination of the two polarities could aid in resolving the degeneracy. That the anti-neutrino run by itself produces slightly larger sensitivity contours is mainly due to the lower cross-section. 
Fig. 4 (Color online) The sensitivity of OPERA for five years of running time in neutrinos (dark curves) and anti-neutrinos (light curves) as well as the combination thereof (colored regions). The sensitivity levels correspond to confidence levels (2 d.o.f.) of $90 \%, 95 \%$, and $99 \%$, respectively
Fig. 5 (Color online) The predicted sensitivity contours of OPERA for different simulated values of $\varepsilon_{\mu \tau}$ ( 2 d.o.f.). The simulated values chosen are $\varepsilon_{\mu \tau}=0$ (upper-left panel), $\varepsilon_{\mu \tau}=-0.3$ (upper-right panel), $\varepsilon_{\mu \tau}=-0.6$ (lower-left panel), and $\varepsilon_{\mu \tau}=-0.3(1+\mathrm{i})$ (lower-right panel). The colored regions correspond to a fit using the correct neutrino mass hierarchy, while the curves are the regions obtained with a fit using the wrong neutrino mass hierarchy. The confidence levels are $90 \%, 95 \%$, and $99 \%$, respectively. This figure only includes the results of running for five years with neutrinos
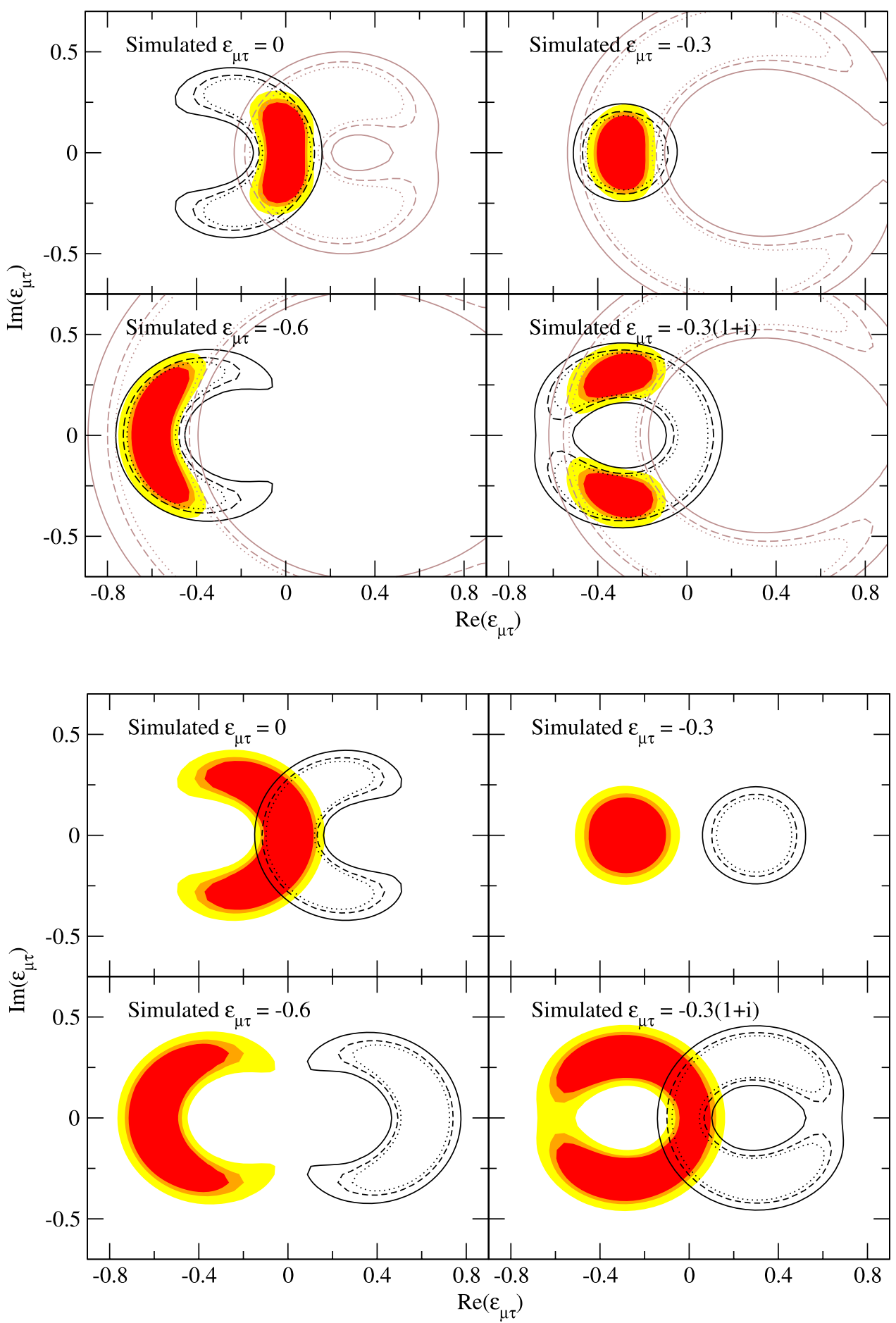

Furthermore, the figure shows the effects of having different simulated values for $\varepsilon_{\mu \tau}$.

In Fig. 5, the effects of fitting the data to the wrong neutrino mass hierarchy are shown. Again, we can observe that the sensitivity contours extend in the direction of a constant number of events, i.e., the circle centered at $\varepsilon_{\mu \tau} \simeq-0.3$. In the case of $\varepsilon_{\mu \tau}=-0.3$, the circle has radius zero and the allowed region is relatively small. This corresponds to the anti-resonance case, where no events are expected. The results of the fit using the wrong neutrino mass hierarchy is a simple mirroring of the result with the correct hierarchy, $\varepsilon_{\mu \tau} \rightarrow-\varepsilon_{\mu \tau}$. This can easily be understood from (6), where a sign change in $\varepsilon_{\mu \tau}$ exactly cancels the sign change in $\Delta m_{31}^{2}$ associated with changing the neutrino mass hierarchy. Strictly speaking, the sensitivity of OPERA to $\varepsilon_{\mu \tau}$ is 
the union of the sensitivities obtained when fitting each mass hierarchy separately.

\section{Summary and conclusions}

We have studied NSIs in connection with the OPERA experiment. Unlike in the previous work by Esteban-Pretel et al. [13], where the focus was on the effective NSI parameters $\varepsilon_{e \tau}$ and $\varepsilon_{\tau \tau}$ due to the external bounds on the other parameters, we have focused on the NSI parameter $\varepsilon_{\mu \tau}$. The reason for this is that $\varepsilon_{\mu \tau}$ is more important for the $\nu_{\mu} \rightarrow \nu_{\tau}$ oscillation probability in OPERA due to the relatively short baseline, as can be seen in our analytic considerations.

We have found that OPERA is actually sensitive to a combination of standard and non-standard parameters, which can easily be observed in (6). The degeneracy in the parameter space, where this combination is constant, is somewhat broken by the energy dependence of the standard term. A much better determination of $\varepsilon_{\mu \tau}$ can be obtained if we consider a five + five year neutrino-anti-neutrino run, especially if compared with a ten year of data taking with neutrinos only, the main reason being the different correlations between standard and non-standard parameters, visible from (6) with the replacement $V \rightarrow-V$ and $\varepsilon_{\mu \tau} \rightarrow \varepsilon_{\mu \tau}^{*}$.

We have also observed that the uncertainty on $\varepsilon_{\mu \tau}$ can be strongly worsened due to our ignorance in the sign of the large mass squared difference $\Delta m_{31}^{2}$, resulting in a reflection of the allowed region for $\varepsilon_{\mu \tau}$ in the imaginary axis. This holds true in the case of running in neutrinos only as well as running with both neutrinos and anti-neutrinos.

By means of the simple result of (6), we found that an anti-resonance occurs when the standard and NSI parameters cancel. In this case, the effective Hamiltonian element $H_{\tau \mu}$ vanishes and no $v_{\mu} \rightarrow v_{\tau}$ events would be observed.

The above analytic considerations were illustrated by our numeric simulations using a modified version of the GLoBES software. In particular, it is evident from Fig. 4 that running the OPERA experiment for five years in each polarity would be much more efficient in constraining the $\varepsilon_{\mu \tau}$ parameter space than running for ten years with neutrinos only.

Finally, we again want to mention that atmospheric neutrino experiments put constraints on $\varepsilon_{\mu \tau}$ which are better than what OPERA is sensitive to [9]. However, these constraints have been computed in a pure two-flavor framework without the interference of $\varepsilon_{e \tau}$. It is known that the bounds on $\varepsilon_{\tau \tau}$ from similar considerations are significantly weakened when extending to a full three-flavor framework. Thus, OPERA will provide a very clean and complementary bound, since only $\varepsilon_{\mu \tau}$ enters into the leading term of the neutrino oscillation probability $P_{\mu \tau}$ due to the short baseline.
Acknowledgements We would like to thank Mark Rolinec and Walter Winter for useful information about the GLoBES software and for the original AEDL file for describing the OPERA experiment.

This work was supported by the Swedish Research Council (Vetenskapsrådet), contract nos. 621-2005-3588 [T.O., M.B.] and 623-20078066 [M.B.], the Royal Swedish Academy of Sciences (KVA) [T.O.], and the Göran Gustafsson Foundation [D.M.]. M.B. and D.M. are grateful to the Royal Institute of Technology (KTH) for kind hospitality during the development of this work.

\section{References}

1. J. Hosaka et al., Super-Kamiokande, Phys. Rev. D 74, 032002 (2006), http://arxiv.org/abs/hep-ex/0604011

2. S.N. Ahmed et al., SNO, Phys. Rev. Lett. 92, 181301 (2004), http://arxiv.org/abs/nucl-ex/0309004

3. N. Oblath, SNO, AIP Conf. Proc. 947, 249 (2007)

4. M.H. Ahn et al., K2K, Phys. Rev. D 74, 072003 (2006), http://arxiv.org/abs/hep-ex/0606032

5. D.G. Michael et al., MINOS, Phys. Rev. Lett. 97, 191801 (2006), http://arxiv.org/abs/hep-ex/0607088

6. S. Abe et al., KamLAND (2008), http://arxiv.org/abs/0801.4589

7. M. Guler et al., OPERA (2000), CERN-SPSC-2000-028

8. R. Acquafredda et al., OPERA, New J. Phys. 8, 303 (2006), http://arxiv.org/abs/hep-ex/0611023

9. N. Fornengo, M. Maltoni, R. Tomàs Bayo, J.W.F. Valle, Phys. Rev. D 65, 013010 (2002), http://arxiv.org/abs/hep-ph/0108043

10. A. Friedland, C. Lunardini, Phys. Rev. D 72, 053009 (2005), http://arxiv.org/abs/hep-ph/0506143

11. T. Ota, J. Sato, Phys. Lett. B 545, 367 (2002), http://arxiv.org/abs/ hep-ph/0202145

12. N.C. Ribeiro et al. (2007), http://arxiv.org/abs/0712.4314

13. A. Esteban-Pretel, P. Huber, J.W.F. Valle (2008), http://arxiv.org/ abs/0803.1790

14. P. Huber, T. Schwetz, J.W.F. Valle, Phys. Rev. D 66, 013006 (2002), http://arxiv.org/abs/hep-ph/0202048

15. T. Ota, J. Sato, N.-A. Yamashita, Phys. Rev. D 65, 093015 (2002), http://arxiv.org/abs/hep-ph/0112329

16. M.C. Gonzalez-Garcia, Y. Grossman, A. Gusso, Y. Nir, Phys. Rev. D 64, 096006 (2001), http://arxiv.org/abs/hep-ph/0105159

17. A.M. Gago, M.M. Guzzo, H. Nunokawa, W.J.C. Teves, R. Zukanovich-Funchal, Phys. Rev. D 64, 073003 (2001), http://arxiv.org/abs/hep-ph/0105196

18. P. Huber, J.W.F. Valle, Phys. Lett. B 523, 151 (2001), http://arxiv. org/abs/hep-ph/0108193

19. J. Kopp, M. Lindner, T. Ota, Phys. Rev. D 76, 013001 (2007), http://arxiv.org/abs/hep-ph/0702269

20. N.C. Ribeiro, H. Minakata, H. Nunokawa, S. Uchinami, R. Zukanovich-Funchal, J. High Energy Phys. 12, 002 (2007), http://arxiv.org/abs/0709.1980

21. M. Campanelli, A. Romanino, Phys. Rev. D 66, 113001 (2002), http://arxiv.org/abs/hep-ph/0207350

22. M. Honda, N. Okamura, T. Takeuchi (2006), http://arxiv.org/abs/ hep-ph/0603268

23. R. Adhikari, S.K. Agarwalla, A. Raychaudhuri, Phys. Lett. B 642, 111 (2006), http://arxiv.org/abs/hep-ph/0608034

24. M. Blennow, T. Ohlsson, J. Skrotzki, Phys. Lett. B 660, 522 (2008), http://arxiv.org/abs/hep-ph/0702059

25. J. Kopp, M. Lindner, T. Ota, J. Sato, Phys. Rev. D 77, 013007 (2008), http://arxiv.org/abs/0708.0152

26. N. Kitazawa, H. Sugiyama, O. Yasuda (2006), http://arxiv.org/ abs/hep-ph/0606013

27. P. Huber, M. Lindner, W. Winter, Comput. Phys. Commun. 167, 195 (2005), http://arxiv.org/abs/hep-ph/0407333 
28. P. Huber, J. Kopp, M. Lindner, M. Rolinec, W. Winter, Comput. Phys. Commun. 177, 432 (2007), http://arxiv.org/abs/ hep-ph/0701187

29. S. Davidson, C. Peña-Garay, N. Rius, A. Santamaria, J. High Energy Phys. 03, 011 (2003), http://arxiv.org/abs/hep-ph/0302093

30. J. Abdallah et al., DELPHI, Eur. Phys. J. C 38, 395 (2005), http:// arxiv.org/abs/hep-ex/0406019

31. E.K. Akhmedov, R. Johansson, M. Lindner, T. Ohlsson, T. Schwetz, J. High Energy Phys. 04, 078 (2004), http://arxiv.org/ abs/hep-ph/0402175
32. M. Komatsu, P. Migliozzi, F. Terranova, J. Phys. G 29, 443 (2003), http://arxiv.org/abs/hep-ph/0210043

33. P. Huber, M. Lindner, M. Rolinec, T. Schwetz, W. Winter, Phys. Rev. D 70, 073014 (2004), http://arxiv.org/abs/hep-ph/0403068

34. M.D. Messier (1999), UMI-99-23965

35. E.A. Paschos, J.Y. Yu, Phys. Rev. D 65, 033002 (2002), http:// arxiv.org/abs/hep-ph/0107261

36. A.M. Dziewonski, D.L. Anderson, Phys. Earth Planet. Inter. 25, 297 (1981)

37. M. Maltoni, T. Schwetz, M.A. Tórtola, J.W.F. Valle, New J. Phys. 6, 122 (2004), http://arxiv.org/abs/hep-ph/0405172 